IGCS20_1052

\section{GLIOMATOSIS PERITONEI ASSOCIATED WITH OVARIAN GERM CELL TUMOURS- A CLINICOPATHOLOGICAL CASE SERIES}

${ }^{1}$ A Jayraj*, 'S Kumar, ${ }^{1} \mathrm{~N}$ Bhatla, ${ }^{2} \mathrm{~S}$ Thulkar, ${ }^{2} \mathrm{~S}$ Thulkar, ${ }^{3} \mathrm{~L}$ Kumar. ${ }^{1}$ Department of Obstetrics and Gynaecology, All India Institute of Medical Sciences, India; ${ }^{2}$ Department of Pathology, All India Institute of Medical Sciences, India; ${ }^{3}$ Department of Medical Oncology, All India Institute of Medical Sciences, India

\subsection{6/ijgc-2020-IGCS.81}

Introduction Gliomatosis peritonei (GP) is a rare condition characterized by the presence of mature glial tissue in the peritoneum of patients with ovarian teratomas. In this study, we investigated the clinicopathological features of ovarian teratoma with GP.

Methods From 2016 to 2019, cases of ovarian teratoma with GP treated at AIIMS, New Delhi were retrospectively analyzed for pathology, treatment, survival and prognostic information.

Results The median age of five patients identified with GP was 27 years. All patients had unilateral ovarian mass with moderate to massive ascites at presentation. One patient had bilateral pleural effusion. The average tumour size was $20 \mathrm{~cm}$. Preoperative serum CA-125 (median:392 IU/mL, 141-882 IU/ $\mathrm{mL}$ ) and alpha-fetoprotein levels (median:1118 ng/mL, 219$2313 \mathrm{ng} / \mathrm{mL}$ ) were elevated. Four patients underwent conservative surgery, with complete cytoreduction. One patient had mature cystic teratoma, one had low grade immature teratoma and three had high grade immature teratoma. All patients had stage 1A-1C disease. Intranodal glial tissue was identified in external iliac lymph nodes of one patient. Postoperatively, all immature teratoma patients received BEP chemotherapy. One patient developed growing teratoma syndrome and underwent secondary cytoreduction. At median follow-up of 30 months, all patients were alive without disease.

Conclusion GP is associated with favorable outcomes. Presence of significant ascites or pleural effusion and elevated CA 125 and alpha-fetoprotein levels preoperatively in patients with ovarian teratoma is suggestive of gliomatosis peritonei. It may be associated with mature or immature teratoma of any grade of differentiation. Adequate sampling to rule out immature elements is necessary for a definitive diagnosis of GP.

\section{IGCS20_1053}

\section{SENTINEL LYMPH NODE IDENTIFICATION IN EARLY STAGE OVARIAN CANCER: IS IT STILL POSSIBLE AFTER PRIOR TUMOR RESECTION?}

P Laven*, R Kruitwagen, S Lambrechts. Maastricht Universitair Medisch Centrum, Netherlands

10.1136/ijgc-2020-IGCS.82

Objective Sentinel lymph node (SLN) detection in ovarian cancer is feasible when tracers are injected before the pathological ovary is resected. This study aims to investigate whether the SLN identification is also feasible in patients whose ovarian tumor has already been resected with injection of the tracer into the ovarian ligaments stumps, i.e. in the event that a frozen section confirms malignancy.
Abstract 87 Table 1 Sentinel nodes found
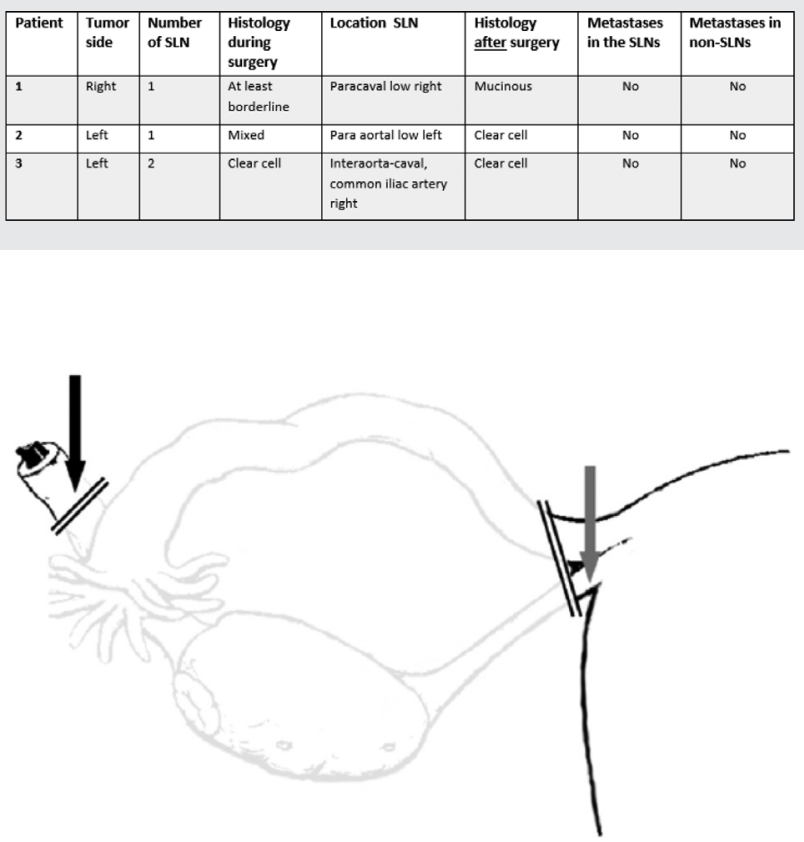

Abstract 87 Figure 1 Location of injection of tracers Tracers were injected on the ventral and dorsal sides of both ligament remains.

Black arrow $=$ remnant of infundibulo-pelvic ligament.

Grey arrow = remnant of the ovarian ligament (proper ovarian ligament).

Methods Patients who underwent laparotomy with frozen section confirming an ovarian malignancy, and those who underwent a second staging laparotomy after prior resection of a malignant ovarian mass, were included. Blue dye and a radioactive isotope were injected in the stumps of the ligamentum ovarium proprium and the ligamentum infundibulopelvicum. After an interval of at least 15 -minutes, the $\mathrm{SN}(\mathrm{s})$ were identified using either the gamma-probe and/or blue dye.

Results A total of 11 patients were included in the study, the sentinel node (SLN) procedure was completed in all 11 patients. At least one SLN was identified in 3 patients, resulting in a rather low detection rate of $27,3 \%$.

Conclusion In this study we showed that SLN procedure after (previous) resection of the tumor seems inferior to detect sentinel nodes when compared to injection of the tracer in the ovarian ligaments before tumor resection.

\section{IGCS20_1054}

\section{DERMATOFIBROSARCOMA PROTUBERANS (DFSP) OF VULVA: CASE REPORT AT PONTIFICIA UNIVERSIDAD CATÓLICA DE CHILE}

E Pertossi* ${ }^{*}$ M Barriga, K García, D Mayerson, A Cuadra, S Gonzalez, P Coronado. Pontificia Universidad Católica de Chile, Chile

10.1136/ijgc-2020-IGCS.83

Introduction DFSP is a rare sarcoma of soft tissues. Most of them of low grade and good prognosis. More frequent in trunk of males. Here we present a case vulvar DFSP. 